\section{Memory retention}

Although most primary effector T cells die during the contraction phase, small numbers survive to become memory cells. In Science, Cheroutre and colleagues link expression of homodimeric CD $8 \alpha$ chains (CD $8 \alpha \alpha)$ to CD 8 memory cell generation. CD $8 \alpha \alpha$ was transiently expressed on a subset of CD8 $\alpha \beta$ T cells after antigen exposure in vitro and in vivo. $\mathrm{CD} 8 \alpha \alpha$ induction correlated with expression of the survival factor $\mathrm{Bcl}-\mathrm{x}_{\mathrm{L}}$ and the memory cell marker IL-7R $\alpha$. Consistently, only the direct progeny of lymphocytic choriomeningitis virus (LCMV)-specific CD8 $\alpha \alpha^{+}$ effector T cells could differentiate into memory T cells. Although CD8 $\alpha$ enhancer-deficient mice could generate LCMV-specific effector cells, IL-7R ${ }^{\text {hi }}$ cells were absent and memory cell generation was impaired. Thus, CD8 $\alpha \alpha$ is important for the initial survival of CD8 memory cell precursors.

$J D K W$ Science 304, 590-593 (2004)

\section{Tec in migration}

Phosphatidylinositol-3 kinase and Zap70 are important in chemokine-mediated signaling and migration. However, the common downstream molecule that is essential for these processes is unclear. Using CXCR4-expressing cells, Takesono et al. in Current Biology and Fischer et al. in the Journal of Biological Chemistry show that the CXCL12 activates the Tec kinase Itk, which in turn controls cell polarization and migration. Itk-deficient mice show impaired $\mathrm{T}$ cell and thymocyte migration in response to several chemokines and homing to peripheral organs. This connection with Tec kinase is similar to that in T cell receptor-mediated signaling, suggesting considerable crosstalk between $\mathrm{G}$ protein- and tyrosine kinase-mediated pathways.

PTL

Curr. Biol. (15 April 2004) doi: 10.1016/S0960982204002660 \& J. Biol. Chem. (26 April 2004) doi: 10.1074/jbc.M312848200

\section{Trimming peptides}

Because cells lack carboxypeptidase activity, proteasomes are thought to be responsible for generating mature $\mathrm{C}$ termini of peptides that bind major histocompatibility complex (MHC) class I molecules. In Immunity, Reits et al. show most of these peptides' C termini are created by tripeptidyl peptidase II (TPPII) activity in vivo. TPPII has both an exopeptidase activity and an endopeptidase, which is activated by peptides more than 15 amino acids long. The products of proteasome action fall into this longer peptide pool and serve as substrates for TPPII. In vivo inhibition of TPPII blocked MHC class I epitope generation, whereas the combination of TPPII and proteasome inhibitors had little additional effect. Thus, TPPII is a key participant in MHC class I epitope generation. Immunity 20, 495-506 (2004)

\section{The importance of being IL-2}

Despite being the hallmark of $\mathrm{T}$ cell activation, the dynamics of interleukin 2 (IL-2) production are unclear. This limitation has prevented a full understanding of $\mathrm{T}$ cell activation and differentiation

Research notes written by Laurie A. Dempsey, Peter T. Lee and Jamie D.K. Wilson. in vivo. In the Journal of Immunology, Sojka et al. use a sensitive cytokine capture assay to show that IL-2 secretion in vivo is rapid but transient and closely correlated with its mRNA expression. The IL-2 response of memory cells is quicker and more robust than that of naive $\mathrm{T}$ cells. Adoptive transfer experiments using lymphopenic, TCR-transgenic or wild-type mice show that the secretion of IL-2 by $\mathrm{T}$ cells is regulated by competition in their environment. This competition is clonally specific and lowers the strength of stimulus available to activate the T cells.

PTL

J. Immunol. 172, 6136-6143 (2004)

\section{T-bet expands its role}

The regulatory factors that direct natural killer (NK) and invariant $\mathrm{V}_{\alpha} 14 \mathrm{~J}_{\alpha} 18 \mathrm{NKT}$ cell end-stage maturation are unclear. In Immunity, Glimcher and colleagues show that T-bet, a transcription factor required for $\mathrm{T}$ helper type 1 lineage differentiation, is also required for $\mathrm{NK}$ and $\mathrm{V}_{\alpha} 14 \mathrm{NKT}$ cell development. T-bet-deficient mice had stem cell-intrinsic defects in the terminal maturation of NK and $\mathrm{V}_{\alpha} 14$ NKT cells, which reduced peripheral cell numbers and diminished effector function. T-bet expression was greatest at late stages of NK cell development and was induced by the NKactivating receptors and cytokines linked to NK activation and/or proliferation. These data therefore suggest T-bet is a key factor regulating the final maturation stages and homeostasis of NK and $\mathrm{V}_{\alpha} 14$ NKT cells.

Immunity 20, 477-494 (2004)

$J D K W$

\section{Switching B cells listen to Bach}

Terminally differentiated B cells secrete antibodies, but additional factors influence whether these cells undergo further immunoglobulin (Ig) gene diversification, such as somatic hypermutation (SHM) and class-switch recombination (CSR). In Nature, Muto et al. identify Bach2, a transcriptional repressor, as influencing whether antigen-activated $B$ cells secrete IgM or initiate CSR and SHM. Bach2-deficient mice had a hyper-IgM phenotype and expressed Blimp-1 and XBP-1 proteins. But Bach2 ${ }^{-1-}$ cells had decreased expression of activation-induced cytosine deaminase (AID). Attempts to reconstitute CSR in Bach2-deficient B cells by AID transfection failed, suggesting that Bach2 regulates additional components of the CSR and SHM program.

Nature (19 May 2004) doi:10.1038/nature02596

\section{Digesting Toll}

Details of how Toll-like receptors (TLRs) might participate in phagocytosis are unclear. In Science, Blander and Medzhitov compared clearance of pathogens with that of apoptotic cells by macrophages and found that signaling through TLR regulates the phagocytic machinery. In the absence of TLR2 and TLR4 or their downstream adaptor molecule MyD88, internalization of bacteria and the subsequent maturation of phagosomes into phagolysosomes are impaired. In contrast, uptake of apoptotic cells and maturation of phagosomes by TLR mutants are comparable to that of wild-type cells. These data suggest that an inducible and a constitutive phagosome maturation pathway exist. The inducible pathway is only activated by phagosome cargo that engages TLRs, which subsequently signal through the kinase p38 MAPK.

PTL

Science 304, 1014-1018 (2004) 